\title{
LOW-ENERGY PARTICLE EVENTS ASSOCIATED WITH SECTOR BOUNDARIES
}

\author{
Z. ŠVESTKA, L. FRITZOVÁ-ŠVESTKOVÁ, and J. T. NOLTE \\ American Science and Engineering, Inc., Cambridge, Mass., U.S.A. \\ and \\ H. W. DODSON-PRINCE and E. R. HEDEMAN \\ McMath Hulbert Observatory, The University of Michigan, Pontiac, Mich., U.S.A.
}

(Received 7 April; in revised form 29 June, 1976)

\begin{abstract}
Onsets of some 40 to 45 low-energy proton events during the years 1957-1969 coincided in time with transits of well-defined sector boundaries across the Earth. These events can be interpreted as long-lived proton streams filling up some of the magnetic sectors, indicating an acceleration of protons which is not associated with typical proton-producing flares. The sharp onsets of these particle streams, as well as a deficiency of flare-associated particle events shortly before the boundary transit, indicate that in some cases magnetic sector boundaries can inhibit transverse propagation of low-energy particles in the solar corona or in interplanetary space.
\end{abstract}

\section{Introduction}

When preparing the Catalog of Solar Particle Events, 195.5-1969 (Švestka and Simon, 1975), we noticed that the onset of some low-energy proton enhancements coincided in time with transits of magnetic sector boundaries across the Earth. Wilcox and Ness (1965) observed three similar cases in the years 1963 and 1964. However, at that time the dates of most sector boundary transits were not known with sufficient accuracy to permit a valid test as to whether or not the few discovered concomitant events were real physical associations or simply chance coincidences. Recently, Svalgaard (1975) published an Atlas of Interplanetary Sector Structure, 1957-1974, which contains a list of transit times of all well-defined magnetic sector boundaries across the Earth during the 13 years covered by our Catalog. Therefore, we now can make a systematic comparison of all the onsets of solar particle events near the Earth with the transit times of sector boundaries.

The results of this comparison are shown in Figure 1. Figure 1a, for proton events, reveals a significant peak on the day of the sector boundary transit, with statistical confidence of $4.2 \sigma$. Figure $1 \mathrm{~b}$, for pure electron events, does not show such an increase, but it indicates a deficiency prior to the sector boundary transit, with lower statistical confidence of $2.1 \sigma$.

\section{Proton Events}

Let us first discuss the proton events, for which the increased occurrence on the zero day clearly represents a real physical association. Many of the proton events listed in the 


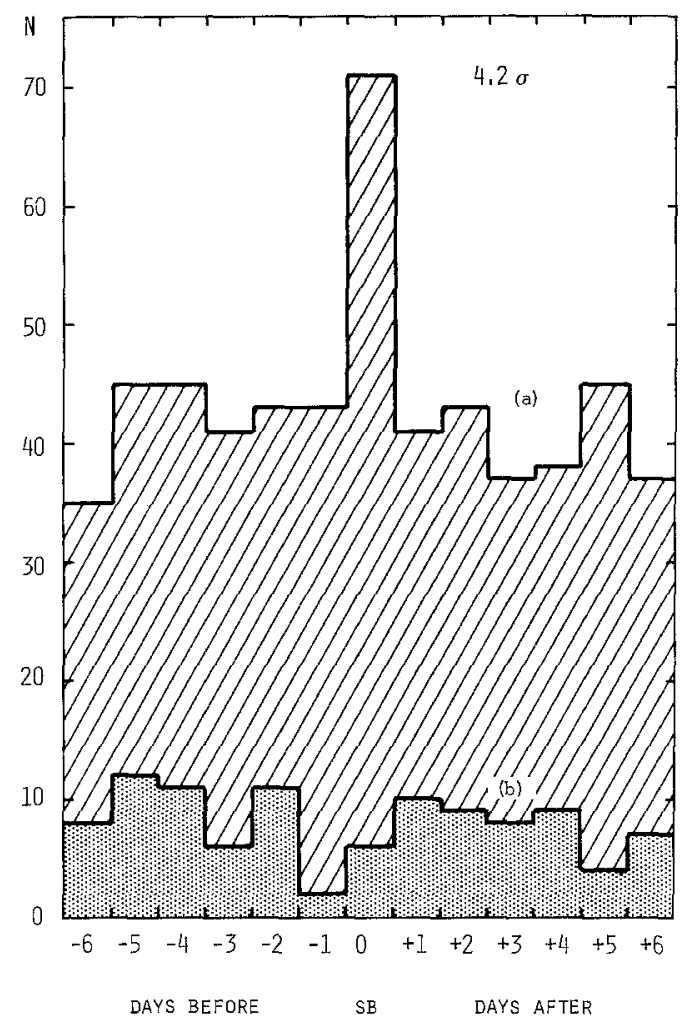

Fig. 1. Number, $N$, of proton events (a) and pure electron events (b) that occurred from -6 days to +6 days around the zero-day when a well-defined sector boundary (SB) passed the Earth. The peak in the occurrence of proton events on the zero day has statistical confidence of $4.2 \sigma$.

Catalog are associated with flares, and this association hardly can be doubted, since the flare sources are distinctly marked by specific 'proton-flare' characteristics. There is no obvious reason why these flare events should start on the day of a sector boundary transit; indeed, if only these events are considered (notations $\boldsymbol{0}, \bigcirc, \mathbf{\square},[$ in the Catalog), there is no increase in the number of cases on the day of a sector boundary transit (fullline histogram in Figure 2). On the other hand, the remaining events, obtained by omitting all flare-associated $(\boldsymbol{\bullet}, \boldsymbol{\odot}, \mathbf{\square}, \boldsymbol{\bullet})$ cases, show the zero peak with greatly increased statistical confidence of $6.1 \sigma$ (dashed histogram in Figure 2).

When the background is subtracted, one can see from Figure 2 that on the days -1 and 0 some 40 to 45 proton events in the Catalog were physically related to transits of magnetic sector boundaries across the Earth. In order to extract them from the whole set of data, we made a list of all the events that started on the zero day. This list still represented a great variety of different kinds of proton enhancements, but one kind seemed to occur at an unusually high rate: a 'diamond' in the Catalog, marking a 'recurrent particle stream' or 'magnetic storminess' (i.e., disturbed geomagnetic 


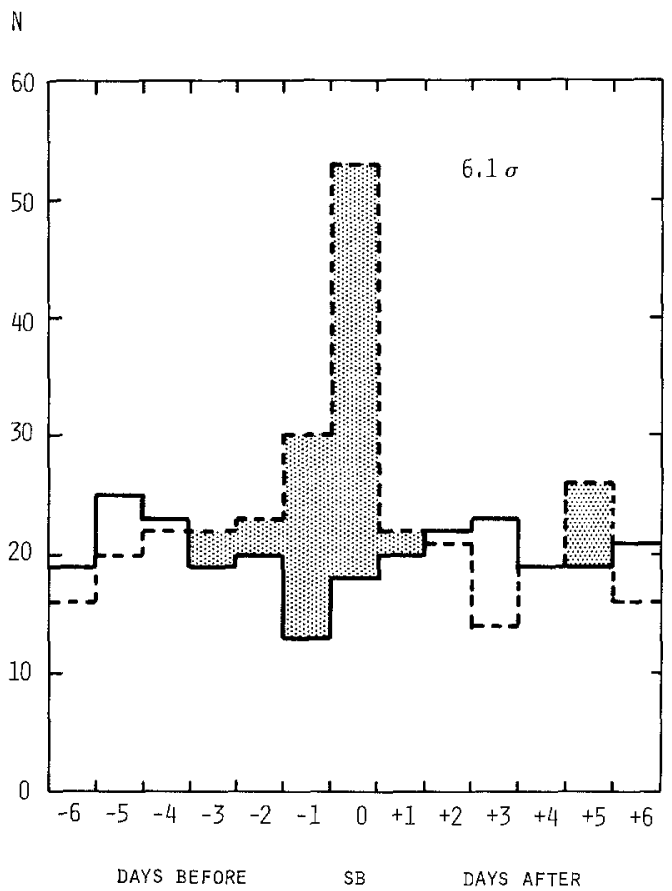

Fig. 2. Same histogram as in Figure la, prepared separately for flare-associated proton events (solid line), and events for which no flare source could be found (dashed line). The dashed-line peak has statistical confidence of $6.1 \sigma$. Note the similarity of the distributions for flare-associated proton events (full line) and electron events (Figure 1b).

conditions, usually recurrent, which may, but need not, be classified as a magnetic storm). Therefore, we have further selected only this particular kind of proton events from the Catalog and the distribution of their onset times relative to sector boundary transits is shown in Figure 3a.

There are several facts which confirm that this criterion properly selected the type of events which produced the zero-day peak in Figures 1a and 2: (1) The statistical confidence of the zero-day peak further increased to $6.5 \sigma$. (2) There is also an increase on the \pm 1 days, which actually is to be expected because of gaps and inaccuracies in the particle records and the one-day time resolution in Svalgaard's Atlas. The statistical confidence of the whole set of coincidences is thus increased to 6.7 $\sigma$. (3) Many of the other events, for which the time interval is $>1$ day (it also may be greater than \pm 6 days) still occurred close to sector boundaries which, though not included in the final Svalgaard's list, can be found in his graphs (some of them are mentioned in the Remarks in Table I).

Table I lists all the proton events of Figure $3 \mathrm{a}$ (i.e., selected through the flare- and diamond-criteria), which occurred on the days $-1,0$, or +1 relative to a sector boundary transit. The list contains 44 events. One can estimate from the excess above 


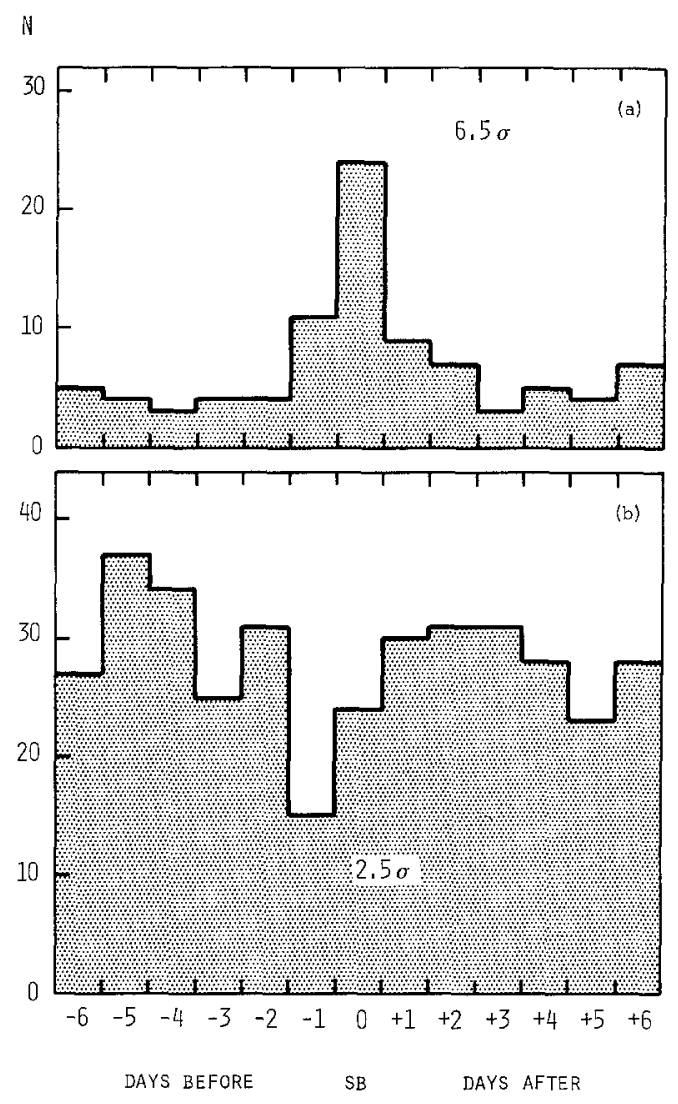

Fig. 3. (a) Same histogram as in Figure 2-dashed line, but prepared only for events marked with a diamond (recurrent particle stream or geomagnetic disturbance) in the Catalog of Solar Particle Events. (b) Sum of the histograms in Figures $1 \mathrm{~b}$ and 2 - full line. It represents all particle events clearly associated with flares. The decrease on the $(-1)$-day has statistical confidence of $2.5 \sigma$.

background in Figure 3 a that $\sim \frac{2}{3}$ of them are real associations, while $\sim \frac{1}{3}$ are chance coincidences. On the other hand, a comparison with Figure 2 indicates that about $\frac{1}{4}$ of the real associations are missing from the table. Thus the filtering is still far from perfect, but we have not been able to make it any better on account of the great inhomogeneities in records of the particle events. A selection of only one homogeneous set of data (e.g., from the IMP satellites) and a study of their records might help to improve the selection of the sector-associated events.

One of the present authors (Švestka, 1968) described an earlier event, on 8 July, 1966, when a sector boundary transit, 30 hours after a major proton flare, coincided with a secondary increase in the particle flux. This increase was very pronounced at energies below $\sim 7 \mathrm{MeV}$, small for energies above $10 \mathrm{MeV}$, and absent for $>20 \mathrm{MeV}$ protons. This also seems to be the case for the events listed in Table I, all of which represent enhancements at low proton energies. Even the PCA events in Table I are very weak 
and, as was the case with the event of $8 \mathrm{July}, 1966$, they may be a result of an expansion of the polar caps to lower latitudes because of geomagnetic disturbances, rather than a penetration of high-energy particles into the polar ionosphere. However, the earlier studied 1966 event was a short-lived increase around and behind the sector boundary, whereas the proton enhancements in Table I generally continue for days and represent long-lasting low-energy particle streams that set in when a sector boundary passes the Earth. Wilcox and Ness (1965) actually discovered three such cases during the preceding solar minimum.

As Table I shows, some of the particle streams followed a flare-associated event that occurred shortly before or, in the same sector, one rotation earlier. For many of the events, however, this does not seem to be the case. Most streams cannot be related to remnants of flare-produced particles in space and some of the proton streams associated with sector boundaries were preceded by periods of several months without any flare-associated particle increase. In some cases a flare could have produced particles on the invisible hemisphere of the Sun, but this hardly could be true for all the events. Thus a question arises regarding the source of these particles.

\section{Discussion}

Let us summarize what we observe: There are certain magnetic sectors in interplanetary space in which the density of low-energy protons is increased. The increased proton flux is first observed when the western boundary of the sector passes across the Earth. After that time the flux remains enhanced for two or more days. In some cases the duration of the enhancement corresponds to the width of the magnetic sector, but generally this is not the rule; the duration is usually shorter, but it can be even longer than the sector width. In some cases the enhancement is observed once again when the sector returns to the Earth after 27 days. More often, however, the proton increase is not recurrent.

In other cases, and these are perhaps most puzzling, the recurrence is intermittent: the enhancement, related to the same boundary, can be seen several times during 10 or more rotations, but there are many rotations in between when increased proton flux is not reported (cf. Table I). Generally, one cannot find any relation of these long-lived proton streams and their intermittent recurrence to energetic proton production in flares so that these streams do not seem to be long-lived remnants of protons accelerated in flares. Therefore, the most plausible explanation appears to be that the low-energy protons causing the enhancements in Table I are accelerated on the Sun through some process which is not associated with flares, or at least not with the major flares that produce strong particle events in space.

The strong association of non-flare proton increases with sector boundary passage implies that in some sense sector boundaries inhibit the propagation of energetic particles across field lines. In order to see effects of this inhibition as proton events, three conditions must be fulfilled: (a) there must be increased production of protons within the considered sector; (b) the western (leading) boundary of the sector must be 
TABLE I

List of proton events associated with transits of magnetic sector boundaries

\begin{tabular}{|c|c|c|c|c|c|c|c|}
\hline Year & $\begin{array}{l}\text { Day of } \\
\text { boundary } \\
\text { transit }\end{array}$ & $\begin{array}{l}\text { Onset } \\
\text { of proton } \\
\text { event }\end{array}$ & Duration & $\begin{array}{l}\text { Onset of a } \\
\text { magnetic } \\
\text { disturbance }\end{array}$ & $\begin{array}{l}\text { Max. } \\
\text { proton } \\
\text { energy }\end{array}$ & $\Delta t^{\mathrm{b}}$ & Remarks \\
\hline 1957 & July 28 & $28^{\mathrm{d}} 15^{\mathrm{h}}$ & $1^{\mathrm{d}}$ & - & weak PCA & $4^{d}$ & (1) \\
\hline \multirow[t]{3}{*}{1960} & Feb. 07 & $07^{\mathrm{d}} 07^{\mathrm{h}}$ & $4^{d}$ & - & weak PCA & $22^{d}$ & $(2)$ \\
\hline & Feb. 28 & $29^{d} 16^{h}$ & $8^{d}$ & $29^{\mathrm{d}}$ & weak PCA & $44^{d}$ & (3A) \\
\hline & July 15 & $14^{\mathrm{d}}$ & $1^{\mathrm{d}}$ & $14^{\mathrm{d}} 17^{\mathrm{h}} 02^{\mathrm{m}}$ & weak PCA & $17^{\mathrm{d}}$ & $(3 \mathrm{~A})$ \\
\hline 1961 & Dec. 01 & $<01^{\mathrm{d}} 03^{\mathrm{h}}$ & $?$ & $01^{\mathrm{d}} 03^{\mathrm{h}}$ & $>3 \mathrm{MeV}$ & $21^{d}$ & (4) \\
\hline 1962 & Feb. 05 & $04^{\mathrm{d}} 12^{\mathrm{h}}$ & $2^{\mathrm{d}}$ & $04^{\mathrm{d}} 09^{\mathrm{h}} 30^{\mathrm{mh}}$ & PCA & $86^{d}$ & \\
\hline \multirow[t]{3}{*}{1963} & May 28 & $27^{\mathrm{d}} 00^{\mathrm{h}}$ & $1^{\mathrm{d}}$ & $27^{\mathrm{d}} 20^{\mathrm{h}}$ & $>10 \mathrm{MeV}$ & $42^{d}$ & $(5,6 \mathrm{~B}, 7)$ \\
\hline & Sept. 14 & $14^{\mathrm{d}} 20^{\mathrm{h}}$ & $?$ & $13^{\mathrm{d}} 19^{\mathrm{h}}$ & weak PCA & $36^{\mathrm{d}}$ & $(6 \mathrm{~B}, 8)$ \\
\hline & Dec. $03^{c}$ & $03^{\mathrm{d}} 08^{\mathrm{h}}$ & $7^{\mathrm{d}}$ & $2^{\mathrm{d}} 21^{\mathrm{h}} 16^{\mathrm{m}}$ & $>0.9 \mathrm{MeV}$ & $36^{\mathrm{d}}$ & $(6 B)$ \\
\hline \multirow[t]{7}{*}{1964} & Jan. $01^{c}$ & $02^{\mathrm{d}} 08^{\mathrm{h}}$ & $5^{\mathrm{d}}$ & $1^{d}$ & $>0.9 \mathrm{MeV}$ & $66^{d}$ & $(6 \mathrm{~B})$ \\
\hline & Jan. 23 & $23^{\mathrm{d}} 07^{\mathrm{h}}$ & $5^{\mathrm{d}}$ & - & $>0.9 \mathrm{MeV}$ & $87^{\mathrm{d}}$ & $(9 \mathrm{C})$ \\
\hline & $\operatorname{Jan} .28^{\mathrm{c}}$ & $28^{\mathrm{d}} 13^{\mathrm{h}}$ & $4^{d}$ & $28^{d}$ & $>0.9 \mathrm{MeV}$ & $92^{d}$ & $(6 \mathrm{~B}, 10)$ \\
\hline & Feb. 19 & $18^{\mathrm{d}} 12^{\mathrm{h}}$ & $10^{\mathrm{d}}$ & - & $>0.9 \mathrm{MeV}$ & $113^{d}$ & $(9 \mathrm{C}, 11)$ \\
\hline & March 04 & $03^{\mathrm{d}} 22^{\mathrm{h}}$ & $4^{d}$ & $3^{\mathrm{d}}$ & $>0.9 \mathrm{MeV}$ & $127^{\mathrm{d}}$ & (12D) \\
\hline & March 31 & $31^{\mathrm{d}} 20^{\mathrm{h}}$ & $2^{\mathrm{d}}$ & - & $>0.9 \mathrm{MeV}$ & $15^{\mathrm{d}}$ & (12D) \\
\hline & Oct. 04 & $<04^{\mathrm{d}} 10^{\mathrm{h}}$ & $>2^{d}$ & $03^{\mathrm{d}} 12^{\mathrm{h}} 43^{\mathrm{m}}$ & $>0.9 \mathrm{MeV}$ & $202^{d}$ & (12D) \\
\hline \multirow[t]{4}{*}{1965} & Apr. 16 & $17^{\mathrm{d}}$ & $4^{\mathrm{d}}$ & $17^{\mathrm{d}} 13^{\mathrm{h}} 12^{\mathrm{m}}$ & weak PCA & $71^{d}$ & $(13 \mathrm{E})$ \\
\hline & June 29 & $30^{\mathrm{d}} 06^{\mathrm{h}}$ & $2^{\mathrm{d}}$ & - & $>0.9 \mathrm{MeV}$ & $17^{\mathrm{d}}$ & \\
\hline & July 06 & $06^{\mathrm{d}} 04^{\mathrm{h}}$ & $2^{\mathrm{d}}$ & $06^{\mathrm{d}} 04^{\mathrm{h}} 50^{\mathrm{m}}$ & $>1.0 \mathrm{MeV}$ & $23^{d}$ & $(13 E)$ \\
\hline & Nov. 25 & $25^{\mathrm{d}} 22^{\mathrm{h}}$ & $5^{d}$ & 一 & $>1.0 \mathrm{MeV}$ & $52^{\mathrm{d}}$ & (14) \\
\hline \multirow[t]{3}{*}{1966} & July 04 & $03^{\mathrm{d}} 22^{\mathrm{h}}$ & $?$ & - & $>0.9 \mathrm{MeV}$ & $9^{d}$ & $(8)$ \\
\hline & Aug. 18 & $18^{\mathrm{d}} 00^{\mathrm{h}}$ & $20^{\mathrm{d}}$ & - & $>0.9 \mathrm{MeV}$ & $20^{d}$ & \\
\hline & Oct. 30 & $31^{\mathrm{d}} 00^{\mathrm{h}}$ & $3^{d}$ & $30^{\mathrm{d}} 12^{\mathrm{h}}$ & $>0.9 \mathrm{MeV}$ & $33^{\mathrm{d}}$ & (15) \\
\hline \multirow[t]{7}{*}{1967} & Jan. 06 & $07^{\mathrm{d}} 14^{\mathrm{h}}$ & $2.5^{\mathrm{d}}$ & $07^{\mathrm{d}} 08^{\mathrm{h}}$ & $>0.9 \mathrm{MeV}$ & $4^{d}$ & (16) \\
\hline & Feb. 07 & $07^{\mathrm{d}} 15^{\mathrm{h}}$ & $?$ & $06^{\mathrm{d}} 16^{\mathrm{h}} 36^{\mathrm{m}}$ & PCA & $5^{d}$ & $(17)$ \\
\hline & March 23 & $24^{d} 14^{h}$ & $3^{\mathrm{d}}$ & - & $>0.9 \mathrm{MeV}$ & $25^{d}$ & (18) \\
\hline & Sept. 27 & $26^{\mathrm{d}} 08^{\mathrm{h}}$ & $2^{\mathrm{d}}$ & - & $>1.0 \mathrm{MeV}$ & $7^{d}$ & $(19 F)$ \\
\hline & Oct. 03 & $03^{\mathrm{d}} 06^{\mathrm{h}}$ & $3^{d}$ & - & $>1.0 \mathrm{MeV}$ & $14^{\mathrm{d}}$ & $(20 G)$ \\
\hline & Oct. 24 & $25^{\mathrm{d}} 14^{\mathrm{h}}$ & $?$ & - & $>1.0 \mathrm{MeV}$ & $18^{\mathrm{d}}$ & $(19 F)$ \\
\hline & Oct. 28 & $27^{\mathrm{d}} 07^{\mathrm{h}}$ & $?$ & $27^{\mathrm{d}} 08^{\mathrm{h}}$ & $>1.0 \mathrm{MeV}$ & $1^{d}$ & $(20 G)$ \\
\hline \multirow[t]{3}{*}{1968} & Jan. 16 & $16^{\mathrm{d}} 08^{\mathrm{h}}$ & $1^{\mathrm{d}}$ & $16^{\mathrm{d}} 15^{\mathrm{h}}$ & $>0.8 \mathrm{MeV}$ & $2^{d}$ & $(19 F, 21)$ \\
\hline & Feb. 11 & $12^{d} 09^{h}$ & $>3^{d}$ & 一 & $>9 \mathrm{MeV}$ & $10^{\mathrm{d}}$ & $(19 \mathrm{~F})$ \\
\hline & Feb. 27 & $26^{\mathrm{d}} 05^{\mathrm{h}}$ & $2^{d}$ & - & $>29 \mathrm{MeV}$ & $9^{d}$ & $(20 \mathrm{G})$ \\
\hline \multirow[t]{5}{*}{1968} & March 10 & $10^{d} 01^{h}$ & $?$ & $09^{\mathrm{d}} 23^{\mathrm{h}} 40^{\mathrm{m}}$ & $>9 \mathrm{MeV}$ & $22^{\mathrm{d}}$ & $(8,19 F)$ \\
\hline & Apr. 06 & $06^{\mathrm{d}} 08^{\mathrm{h}}$ & $2^{d}$ & $05^{\mathrm{d}} 13^{\mathrm{h}} 28^{\mathrm{m}}$ & $>9 \mathrm{MeV}$ & $7^{\mathrm{d}}$ & $(19 F)$ \\
\hline & Aug. 05 & $05^{\mathrm{d}} 11^{\mathrm{h}}$ & $>1^{d}$ & $05^{\mathrm{d}}$ & $>29 \mathrm{MeV}$ & $2^{d}$ & $(20 \mathrm{G})$ \\
\hline & Oct. 16 & $17^{\mathrm{d}} 02^{\mathrm{h}}$ & $>3^{\mathrm{d}}$ & - & $>9 \mathrm{MeV}$ & $13^{d}$ & $(22)$ \\
\hline & Dec. 23 & $23^{\mathrm{d}} 20^{\mathrm{h}}$ & $?$ & - & $>10 \mathrm{MeV}$ & $18^{d}$ & $(8,23 \mathrm{H})$ \\
\hline \multirow[t]{6}{*}{1969} & Jan. 24 & $24^{\mathrm{d}} 04^{\mathrm{h}}$ & $?$ & $24^{\mathrm{d}} 12^{\mathrm{h}}$ & $>9 \mathrm{MeV}$ & $7^{d}$ & $(8,23 \mathrm{H})$ \\
\hline & May 08 & $08^{\mathrm{d}} 19^{\mathrm{h}}$ & $>4^{\mathrm{d}}$ & - & $>9 \mathrm{MeV}$ & $3^{d}$ & $(23 \mathrm{H})$ \\
\hline & May 13 & $13^{d} 05^{h}$ & $6^{\mathrm{d}}$ & $12^{\mathrm{d}} 18^{\mathrm{h}}$ & $>6 \mathrm{MeV}$ & $8^{d}$ & \\
\hline & June 15 & $16^{\mathrm{d}} 06^{\mathrm{h}}$ & $>5^{\mathrm{d}}$ & $16^{\mathrm{d}} 06^{\mathrm{h}}$ & $>0.6 \mathrm{MeV}$ & $5^{d}$ & $(24 J)$ \\
\hline & Sept. 30 & $30^{\mathrm{d}} 12^{\mathrm{h}}$ & $6^{d}$ & in progress & $>1.0 \mathrm{MeV}$ & $3^{\mathrm{d}}$ & $(24 J)$ \\
\hline & Nov. 26 & $25^{\mathrm{d}} 16^{\mathrm{h}}$ & $>5^{\mathrm{d}}$ & $26^{\mathrm{d}} 15^{\mathrm{h}} 07^{\mathrm{m}}$ & $>1.0 \mathrm{MeV}$ & $1^{\mathrm{d}}$ & $(24 J)$ \\
\hline
\end{tabular}


Remarks:

a Hour and minute means sudden commencement. Hour only (or day only) means gradual onset.

${ }^{b} \Delta t$ is the time delay after the latest recorded flare-associated proton event.

c Events discussed by Wilcox and Ness (1965).

(1) Recurrent with PCA on July 1, when the boundary was not well-defined.

(2) Recurrent with flare-associated PCA on January 11 and sc-associated PCA on January 13; the boundary transit was on January 13.

(3A) The same boundary A.

(4) This may be a recurrent event with strong proton event on November 10 , which was seen on the western limb.

(5) Flare with types II + IV occurred $32^{\mathrm{h}}$ earlier at $85 \mathrm{~W}$.

(6B) The same boundary B.

(7) Recurrent with events on Feb. 9, March 8, April 4, May 1, and June 25; the boundary had a complex structure on these dates.

(8) Another event set in when this event was still in progress.

(9C) The same boundary C.

(10) Flare imp. 2 occurred $48^{\mathrm{h}}$ earlier at $08 \mathrm{~W}$.

(11) Recurrent event occurred on March 16, when the boundary had a complex structure.

(12D) The same boundary D.

(13E) The same boundary E.

(14) This boundary was newly formed in rotation 1807. Prior to the Nov. 25 event it might have been associated with similar events on Sept. 1 and 29 ; later on with the event on Dec. 24.

(15) Pioneer 7 gives $30^{\mathrm{d}} 12^{\mathrm{h}}$ as onset time for $>0.6 \mathrm{MeV}$ protons. One rotation before Pioneer 7 observed a complex structure in $>0.6 \mathrm{MeV}$ proton flux, starting on $3^{\mathrm{d}} 22^{\mathrm{h}}$ (boundary transit $4^{\mathrm{d}}$ ) and lasting until $12^{\mathrm{d}}$ (next boundary transit).

(16) Flare of unknown importance $8^{\mathrm{h}}$ earlier at $77 \mathrm{~W}$. A newly formed boundary.

(17) Two flare-associated particle events occurred 27 days ago (on January 11).

(18) Also PCA, 0.9 dB, but its onset time and duration are not known.

(19F) The same boundary F.

(20G) The same boundary G.

(21) Two flares with type II occurred $36^{\mathrm{h}}$ and $32^{\mathrm{h}}$ earlier at $40 \mathrm{~W}$ and $45 \mathrm{~W}$.

(22) Another proton event without known flare source was associated with the same boundary 27 days later, on November 13.

$(23 \mathrm{H})$ The same boundary H. Similar events, apparently associated with the same boundary, occurred on November 25, when the sector boundary had a complex structure, and on March 17, when the sector narrowed to only a 2 -day width.

(24J) The same boundary J.

strong enough to inhibit (or slow down) transverse particle propagation towards the west; and (c) at least at some distance from the Sun the transverse mode of propagation must be fast enough to distribute the protons over the sector width. The intermittent recurrence of some events listed in Table I would then stem from the fact that these conditions are not fulfilled at all times. In particular, the magnetic definition of the boundary may vary markedly. If the boundary is distinct and sharp, the discontinuity in proton flux is easily recognized and is classified as an 'event'. If the boundary is smeared, the increase in particle flux is gradual and either it is not classified as a 'proton event' at all, or the onset time is given with great uncertainty.

One possibility is that the inhibition (b) occurs in interplanetary space. Let us suppose that in one particular magnetic sector the non-flare production of protons is 
significantly higher than in the neighbouring sectors. The protons spread out through diffusion and despite the fact that diffusion across the field lines is slow, in long term the protons fill the sector. The transverse diffusion is further slowed down wherever the magnetic field is strengthened. This may be the case at well-defined sector boundaries where the magnetic field strength in some cases distinctly increases (cf. Švestka, 1968). Therefore, the boundary represents an obstacle for the diffusing protons, the protons accumulate at the boundary and the population distribution shows a discontinuity there, defined as a 'permanent particle stream onset'.

It is rather doubtful, however, that condition (c) is fulfilled far from the Sun. Roelof and Krimigis (1973), in a detailed analysis of coronal and interplanetary observations from three solar rotations, have found that 'Low-energy solar charged particles cannot cross interplanetary field lines to any measurable extent, and there is no indication of significant "random walking" of field lines'. Therefore, if they are right, the effects of the sector boundary on particle propagation must occur close to the Sun. Hundhausen (private communication) has suggested that most of the solar wind, at least during the declining phase of the solar cycle, comes from coronal holes. These holes lie over relatively small areas of photospheric field, covering less than one-fifth of the solar surface. If this is true not only for the years of declining activity but also throughout the rest of the cycle, then neighboring interplanetary sectors originate from widely spaced photospheric regions. This does not contradict the results recently published by Svalgaard et al. (1975), because their maps do not show which parts of the large-scale fields are closed or open. Thus, if most of the propagation of energetic particles across magnetic field lines occurs fairly low in the solar corona, it is quite likely that different sectors may contain greatly different energetic particle populations.

An additional implication of this interpretation is that the increases would be better associated with particular solar wind sources than with magnetic sectors. As Hundhausen (1972) points out, solar wind streams are well-correlated with magnetic sectors, but some sectors contain two streams. This may be a part of the explanation why some sector boundaries are not associated with particle increases and why some low-energy non-flare events are not associated with sector boundaries.

If proton enhancements are confined within sector boundaries, a sudden drop in proton flux should occur as well, when the eastern (following) boundary passes the Earth. Such cases cannot be checked from the material used for this study, since flux decreases are not classified in the Catalog as particle events. Rapid decreases have been observed, however (Wilcox, 1976), and multiple-spacecraft observations have been used to demonstrate that several decreases of this type are convected in the solar wind (Roelof and Krimigis, 1973).

\section{Flare-Associated Events}

When we plotted separate histograms for proton events associated and not associated with flares in Figure 2, the flare-associated events (full-line histogram) did not show a 
peak on the zero day. However, the histogram indicates a decrease on the day prior to the sector boundary transit, very similar to the one found for electrons in Figure 1b. When both of these histograms are added together, we get the graph in Figure $3 \mathrm{~b}$. The quite pronounced decrease on the $(-1)$-day has a statistical confidence of $2.5 \sigma$.

All the particle events in Figure $3 b$ were clearly associated with flares. If a strong sector boundary lies between the Earth and the sector in which the flare occurred, the accelerated low-energy particles may be inhibited in their propagation towards the Earth in a manner similar to that of the long-lasting particle streams. About 4-5 days prior to the boundary transit the boundary foot-line crosses the central meridian of the Sun. Particles from some events, situated more to the east and occurring at this time, may not reach the Earth. This deficiency should increase as the boundary approaches the Earth, reaching maximum on the $(-1)$ day, when only particles from events more than $\sim 50^{\circ}$ to the west have perfectly free access to the Earth. As soon as the boundary passes the Earth, the access for all particles from flares to the east of $\sim 60^{\circ} \mathrm{W}$ is open and only particles coming from sources more to the west can be inhibited in their propagation to the Earth.

One can see that Figure $3 b$ is in good qualitative agreement with these expectations. However, these expectations are no more than a greatly simplified model, since the propagation of flare-associated particles is a rather complex process. In some cases low-energy particles not only diffuse, but also travel rapidly across long distances in the solar corona before being released into space (e.g., Palmer and Smerd, 1972; Cherki et al., 1974); in interplanetary space the magnetic field structure can deviate from the ideal simple configuration of the Archimedes spiral (e.g., Keath et al., 1971); and, as we saw in the preceding section, only a few boundaries are expected to produce the inhibiting effect. Thus we can expect (and observe) only a statistical trend in the data, showing a reduction in the number of flare particle events just prior to sector boundary passage.

\section{Acknowledgements}

This work was supported at AS\&E by NASA Contract NAS 8-27758. One of the authors (J.T.N.) has benefited from his participation in the Skylab Solar Workshop Series on Coronal Holes. The Workshops are sponsored by NASA and National Science Foundation and managed by the High Altitude Observatory (NCAR). We are thankful to Dr S. Kahler of AS\&E for drawing our attention to similar cases discussed eleven years ago, and to Dr J. M. Wilcox for a helpful discussion.

\section{References}

Cherki, G., Mercier, J. P., Raviart, A., Treguer, L., Maccagni, D., Perotti, F., and Villa, G.: 1974, Solar Phys. 34, 223.

Hundhausen, A. J.: 1972, Coronal Expansion and Solar Wind, Springer Verlag, New York.

Keath, E. P., Bukata, R. P., McCracken, K. G., and Rao, U. R.: 1971, Solar Phys. 18, 503.

Palmer, I. D. and Smerd, S. F.: 1972, Solar Phys. 26, 460. 
Roelof, E. C. and Krimigis, S. M.: 1973, J. Geophys. Res. 78, 5375.

Svalgaard, L.: 1975, Stanford University Institute for Plasma Research, Report No. 629.

Svalgaard, L., Wilcox, J. M., Scherrer, P. H., and Howard, R.: 1975, Solar Phys. 45, 83.

Švestka, Z.: 1968, Solar Phys. 4, 361.

Švestka, Z. and Simon, P. (eds.): 1975, Catalog of Solar Particle Events, 1955-1969, D. Reidel Publ. Co., Dordrecht and Boston.

Wilcox, J. M.: 1976, private communication.

Wilcox, J. M. and Ness, N. F.: 1965, J. Geophys. Res. 70, 5793. 